\title{
PROCESS MINING IMPLEMENTATION BENEFITS FOR ORGANIZATIONS USING ERP SYSTEMS
}

\author{
Effah A. Kadasah \\ Master Student, King Saud University, Riyadh, Saudi Arabia
}

\begin{abstract}
One of the most valuable assets of an organization is its organizational data. Enterprise recourse planning (ERP) is one of the information systems that can be a source for data and implemented processes for the most of the organizations. Business process management and data driven approach are combined in process mining to address the increasing needs of organizations. The core idea of process mining is to utilize ERP or any other information systems implicit data by using algorithms to create new process model, analyze a process model or enhance an existing process model. In this review paper, I will discuss how organizations benefit more from ERP in improving their business process by using process mining technique in business process management cycle. The paper contains examples of related work that use process mining in different business process that have been implemented in ERP.
\end{abstract}

\section{KEYWORDS}

ERP, Process Mining, Business Process Management

\section{INTRODUCTION}

Process mining is as one of leading innovations at business process management (BPM) field in 2012. IEEE has determined a Task Force who dedicated about process mining which has been lead by Wil van der Aalst, from Eindhoven University of Technology, The Netherlands (Van Der Alast et al., 2011). This task force published a Process Mining Manifesto where the process mining has been introduced as a research field which contains the main concepts description, principles guidance and related challenges (Van Der Alast et al., 2011).

Business process mining is a young research topic that related to both machine learning and data mining on area and process modelling and analysis on the area (Van Der Alast et al., 2011). ERP is a strategic tool which synchronizes, integrates, and streamlines data and processes of the organization into one single system to gain a competitive edge in the uncertain business environment (Madanhire \& Mbohwa, 2016). The implementation projects of ERP systems are frequently considered as failure due to several reasons include: inherent complexity, resource intensity, required financial investment, required time, management challenges and risks and number of employees involved (Fleig, 2018). Chistian fleig and Dominik Augesnstein found out that the activities of doing the business process transformation and re-engineering are necessary in the actual ERP implementation or during the improvement of business process within the system (Fleig, 2018).

Ana Pajić and Dragana Bečejski mentioned that the organizations utilize Enterprise Resource Planning (ERP) systems to integrate the data and processes of organization into single database where those systems are handling a huge amount of data related to the actual execution of business processes and the goal is to discover from transaction log a model of how the business processes are actually completed (Pajić, \& Bečejski, 2016). The main idea of business process mining is to extract the execution of business processes preserved in event logs which are available in information system like ERP in order to directly build the models of business processes, compare existing business process models with the new automatically constructed models to identify deviations and bottlenecks and to enhance the business processes (R'bigui \& Cho, 2017).

This paper is organized as follows. Section 2 is presenting a brief description of an ERP system with related modules and details about BPM lifecycle phases. Section 3 contains the description of process mining and its related concepts, it's also shows how the process mining techniques can be implemented in each 
phase of BPM cycle. In section 4, the related works are discussed by presenting three examples that use process mining in different business processes that have been implemented in ERP. Also, in the same section which is section $4 \mathrm{I}$ list the benefits the organizations gain from implementing process mining using their ERP. Concluding remarks are given in section 6.

\section{ERP \& BPM}

According to American Production and Inventory Control Society, ERP is defined as a "method for the effective planning and controlling of all resources needed to take, make, ship and account for customer orders in a manufacturing, distribution or service organization" (Madanhire \& Mbohwa, 2016). Ignato Madanhire and Charles Mbohwa list the basic modules which are consist in ERP as followed: production planning module, purchasing module, ERP inventory control module, sales module, marketing module, ERP financial module and human resources module (Madanhire \& Mbohwa, 2016).

Izzeideen Alomari et al., researchers from University of Kebangsaan in Malysia, states that the ERP is playing an important role in supporting procedures of business by carrying necessary business process information and create changes on the activities of the business (Alomari et al., 2019). They mentioned that ERP is linked to business process highly by which the business process is a collection of interconnected activities that provide output of greater value than the inputs through one or more transference (Alomari et al., 2019).

There are various of definitions about process. Edyta Brzychczy mentioned that according to Grajewski, the process is defined as "a chain of sequential activities that transform measurable inputs (materials, information, people, devices, methods) into a measurable output (products, services, information)" (Brzychczy. 2017).

She considered that the major stage for process management in any organization is their identification which leads to process architecture development and presents a set of particular processes along with the sopecified relations between them (Brzychczy. 2017). Edyta Brzychczy and presents the BPM cycle (Figure 1) on her paper (Brzychczy. 2017). The BPM cycle includes the following phases: process modeling, process analysis, implementation, and process monitoring and controlling.

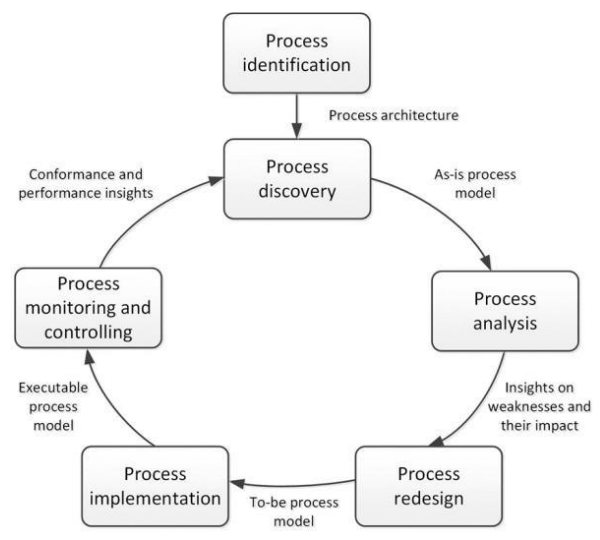

Figure 1. BPM Cycle (Brzychczy. 2017) [Source: http://bpmcenter.org/]

Process modelling (discovery) which involve the development of a process model which is an "as-is" that maps process realization in the organization (Szelągowski, 2018). Process analysis, this stage contains the identification and discovery of the problems related to the emerging process that have been identified and all documents related to it and procedures for the efficiency of the process. The result of this activity is that the organization obtains a set of problems associated with this process and is arranged according to their impact on the organization's results (Szelągowski, 2018). Process redesign which used for identifying the process changes that will support solving the problems that stand out and help the organization to accomplish the desired effects. The result of this phase is a process model of "to-be" process (Szelągowski, 2018). Process implementation, this phase involves the implementation of the resulted process from the process redesign phase (Szelagowski, 2018). Process monitoring and control contains the activity of data analysis during the 
implementation of the process in order to identify if the changes have produced the expected impacts and if the assumed objectives were achieved or not (Szelągowski, 2018).

\section{PROCESS MINING}

Process mining is a technique used in process management which can help the users to produce business processes in an objective manner by analyzing business data which can be extracted from event logs and visualize the actual process flows automatically (Park \& Kang, 2016). The most suitable definition of process mining is the one that has been given in the Process Mining Manifesto: "The idea of process mining is to discover, monitor and improve real processes (i.e., not assumed processes) by extracting knowledge from event logs readily available in today's (information) systems. Process mining includes (automated) process discovery (i.e., extracting process models from an event log), conformance checking (i.e., monitoring deviations by comparing model and log), social network/organizational mining, automated construction of simulation models, model extension, model repair, case prediction, and history-based recommendations." (Van Der Alast et al., 2011). (Figure 2) displayes process mining prerequisites (Van Der Alast et al., 2011).

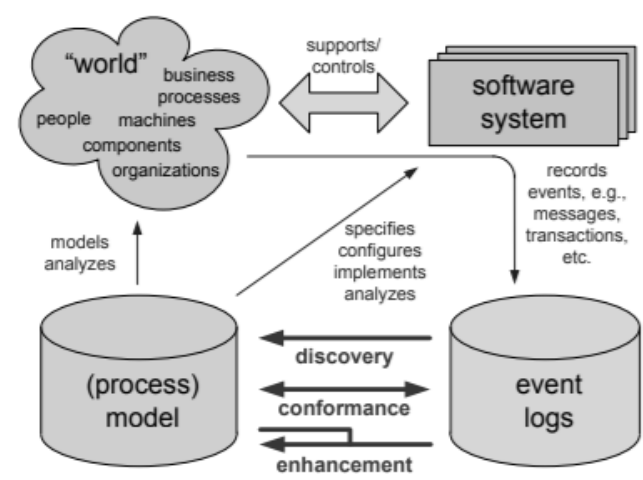

Figure 2. Process Mining Context (Van Der Alast et al., 2011)

\subsection{Process Mining Concepts}

Event can be defined as an activity corresponding to the starting point of the process mining, the sequential relation between events is required in process mining techniques where each activity is a unique process instance, i.e. it belongs to a specific event (Van Der Alast, 2012). Regarding what event log are actuating storing Dusanka Dakic et al., states that an event log are storing cases and activities information and also event performers information (like persons or devices), event timestamps or other recorded data of any of an event element (Dakic et al., 2018).

Dusanka Dakic et al., describe 3 process mining types: discovery, conformance and enhancement. Discovery: this technique is using to produce new process model based on data generated from event log, without depending on any prior information about the new process (Dakic et al., 2018). Conformance: this technique used to compare between an existing process model and data generated from event log of the same process which can be used to review the results of this process if it's exactly the same as recorded in event $\log$ (Dakic et al., 2018). Enhancement: this technique can be used to improve an existing process model depending on the actual process information which has been recorded in event log and the purpose is to change or a prior model (Dakic et al., 2018).

Van der Aalst et al., defined three different perspective that may covered by process mining which are process, organizational and case perspective. Process perspective which focuses on the control flow and the activities ordering. The aim of this perspective is to find the best description of all possible paths by using a Petri net or Event-driven Process Chain (EPC) (Van Der Alast, 2007). Organizational perspective which focuses on the originator field, the involvement of performers and relationship between them. The aim is to frame the organization by perform people classifications in terms of their roles and organization's units or to present relations between each one of the performers (Van Der Alast, 2007).Case perspective which focuses 
on properties of cases. Cases can be distinguished in the process by their path the process or they can be distinguished by the one who is working on a case. Moreover, cases can also be differentiated by the values of the identical data elements (Van Der Alast, 2007).

There are many of process mining software tools which are available for use. Aruna Devi et al., discussed on details about the software tools used for process mining which are ProM tool that developed at Eindhoven University of Technology, Disco which is a complete process mining software that offered by Fluxicon, Celonis Discovery which is a process mining tool provided by Celonis, Fujitsu Process Analytics, Rapid Mine, Process Gold Enterprise Platform and more (Devi, 2006). Dusanka Dakic et al., mentioned that there are 3 main types of process mining algorithms which are Deterministic algorithms (example of this is Alpha algorithm), Heuristic algorithms and Genetic algorithms (Dakic et al., 2018).

\subsection{Using of Process Mining Techniques in BPM Cycle}

Edyta Brzychczy presents the possibilities of using process mining techniques in the BPM cycle where she mentioned that the analysis can be performed using any of the process mining tools like ProM, Celinos or another tool (Brzychczy. 2017). Process modeling (discovery): there are several of algorithms that have been developed which can used to the construct process models based on event logs, examples of those algorithms are: Inductive Miner, Alpha Miner, Heuristic Miner (Brzychczy. 2017). Process analysis: in terms of process analysis, there are several useful techniques that can be used to visualize the event log like: dotted chart, Case flow visualization, Work hand-over graph (Brzychczy. 2017). Process implementation: contains the using of notation to draw and write executable process models like using the BPMN notation (Brzychczy. 2017). It contains the details of events types, activities, executing units and sources of data which can be applied in the organization after the phase of redesign (Brzychczy. 2017). Process monitoring and controlling: it is important in this phase to notice the differences between the realization of the process and the specified process model and the reference to process variation appears in this phase to enable the organization to take a corrective action (Brzychczy. 2017).

\section{RELATED WORKS}

This section shows a summary of several papers found that present an actual case studies related to implement process mining on organizations using ERP systems on some of their modules like financial module and purchasing module. These papers present on details how the data from ERP extracted and processed using process mining techniques and support the decision makers on developing a new business process, do enhancement in an existing process, or support on decision making for individualize or standardize the business process for organizations. First, I will discuss about two case studies from two different resources which present an actual implementation of process mining technique in two different business processes, first one for "purchase to pay" process and second one for "financial" process. In the second sub heading, I will list the benefits that the organizations gain from implementing process mining using their ERP data in improving the business process followed by the organization.

\subsection{Examples of Implementing Process Mining on ERP}

Christian Fleig, Dominik Augenstein and Alexander Maedche from Karlsruhe Institute of Technology in Germany studied an actual case study for a manufacturing corporation to demonstrate how process mining can be used for process decision making in an SAP S/4 HANA implementation project (Fleig, 2018). The corporation implements process mining for the analysis of the purchase to pay "Purchasing" process and the order to cash "Sales" processes to specify whether the new "to be" process should be standardized or to be individualized (Fleig, 2018). They described how the process mining can be used for business processes comparison and their variants against the different standards by following three steps which are: first, decide which standard is a nominee for implementation; second, contains the implementation of required deviations from the standard; third, contains the estimation of the impacts on an organization (Fleig, 2018). They found out that the use of process mining allows all variants to be considered in the decision to determine whether to individualize or standardize the process (Fleig, 2018). They mentioned that the managers from the 
corporation stated that process mining project helped them in utilizing BPM as an essence capability of their organization and to increase the "process oriented thinking" of themselves and their employees (Fleig, 2018).

Michael Werner from Auckland University of Technology in New Zealand discussed about how process mining can be used as an incoming technique for data analysis to support auditors in the context of financial and accounting structure (Werner, 2017). In his research, he investigates how process mining can be used to improve process audits that are an important part of financial audits where It focuses on source data from ERP systems and investigates how this data can be used in a novel way to produce less complex process models that provide precise information on the control flow from an accounting perspective (Werner, 2017).

Mahendrawathi ER, Shania Zayin and Firman Pamungkas promotes on their paper the use of process mining and root cause analysis from BPM field to make post implementation review of ERP implementation at the level of business process (Mahendrawathi et al., 2017). The case study company is a chemical company that is changing their old ERP system with SAP ERP. They presented how the event log for the process of procurement is generated from the activities data stored in SAP database (Mahendrawathi et al., 2017). They used the Disco tool to process the event log to conduct more analysis where they applied the fuzzy miner to discover the process model from event log (Mahendrawathi et al., 2017). They present that process mining helped them find out the bottleneck appears which related to technical issues, data migration issues and cultural issues like high dependency of hard copy approval (Mahendrawathi et al., 2017). Those results that they found helped them figure out the issues that they need to resolve so the company can realize the operational efficiency benefit and eventually long-term benefit of ERP implementation (Mahendrawathi et al., 2017).

\subsection{Benefits of Process Mining for Organizations}

Aruna Devi et al., list the benefits of process mining in four points. First, they considered that the objectivity is the main benefit of process mining because it helps the organizations to produce better process models for good productivity (Devi, 2006). Second, they mentioned that the time is major constraint in performing tasks, where the process mining techniques provide faster results if there is an accurate data used (Devi, 2006). Third, they notice that for any organization specially for the manufacturing industries, process mining offers the potential to find exceptions and allows to do conformance checking, so that it can avoid any deviation in production (Devi, 2006). The last benefit introduced by them is that the process mining has the ability to produce different opinions on the same data set (Devi, 2006).

\section{RESULT}

As a result of this review paper, the following (Figure 3) shows the relationship between the three main topics that I've been discussed about on this paper which are business process management (BPM), enterprise resource planning (ERP) and process mining.

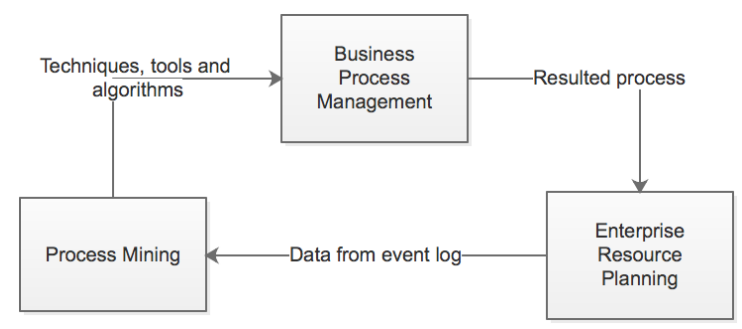

Figure 3. The relationship between BPM, ERP and process mining

The BPM produce a resulted process to be implemented in ERP as a new process or an enhancement in an actual process. Where from the ERP system we can extract the needed data from event logs that stored in the system as a history of people, data, time, and activities accomplished related to a specific process. The data that has been extracted from ERP are used in process mining by implementing their tools, algorithms and techniques to find the issues and gaps related to those data and processes by following the steps and phases of BPM lifecycle including: process discovery, process analysis, process redesign, process implementation and 
process monitoring and controlling. That step helps the high management or decision makers to make a decision on implementing a new process or an applying an enhancement in an actual process so this resulted business process can be again implemented in ERP system.

\section{CONCLUSION}

The goal of this paper is to gather information about how the organizations can benefit from ERP system data which can be extracted from event log and implement process mining techniques using extracted data from ERP in the phases of BPM cycle to came up with new process, or an enhancement for an existing process, or support on decision making for individualize or standardize the business process for an organization. The paper presented the relationship between the three main topics which are business process management, enterprise resource planning and process mining.

The three main theory backgrounds have been described in 3 separated sections. The business process management's role is to give context and setting for overall business process development and how the process mining techniques can be used through the BPM life cycle. Therefore, ERP systems act as a vital source of data, and where the improved process can be implemented. Finally, process mining context, is responsible of providing the analytical work to make the findings from ERP data to improve business processes.

\section{REFERENCES}

Alomari, I. A., Amir, A. M., Aziz, K. A., \& Auzair, S. M. (2019). Enterprise resource planning system business process attributes: A research note. IJAR, 5(3), 111-115.

Brzychczy, E. (2017). Use of Process Mining Techniques in an Enterprise. Inzynieria Mineralna-Journal of the Polish Mineral Engineering SocietY, (2), 237-244.

Dakic, D., Stefanovic, D., Cosic, I., Lolic, T., \& Medojevic, M. (2018). Business Process Mining Application: A Literature Review. Annals of DAAAM \& Proceedings, 29.

Devi, A. T. (2006). An informative and comparative study of process mining tools. Int. J. Sci. Eng. Res, 8(5), 8-10.

Fleig, C., Augenstein, D., \& Maedche, A. (2018). Process Mining for Business Process Standardization in ERP Implementation Projects-An SAP S/4 HANA Case Study from Manufacturing. In BPM (Dissertation/Demos/Industry) (pp. 149-155).

Madanhire, I., \& Mbohwa, C. (2016). Enterprise resource planning (ERP) in improving operational efficiency: Case study. Procedia CIRP, 40, 225-229.

Mahendrawathi, E. R., Zayin, S. O., \& Pamungkas, F. J. (2017). ERP post implementation review with process mining: A case of procurement process. Procedia Computer Science, 124, 216-223.

Pajić, A., \& Bečejski-Vujaklija, D. (2016). Metamodel of the artifact-centric approach to event log extraction from ERP systems. International Journal of Decision Support System Technology (IJDSST), 8(2), 18-28.

Park, S., \& Kang, Y. S. (2016). A study of process mining-based business process innovation. Procedia Computer Science, 91, 734-743.

R'bigui, H., \& Cho, C. (2017). The state-of-the-art of business process mining challenges. International Journal of Business Process Integration and Management, 8(4), 285-303.

Szelągowski, M. (2018). Evolution of the BPM Lifecycle. In Federated Conference on Computer Science and Information Systems (pp. 205-211).

Van Der Aalst, W. M., Reijers, H. A., Weijters, A. J., van Dongen, B. F., De Medeiros, A. A., Song, M., \& Verbeek, H. M. W. (2007). Business process mining: An industrial application. Information Systems, 32(5), 713-732.

Van Der Aalst, W., Adriansyah, A., De Medeiros, A. K. A., Arcieri, F., Baier, T., Blickle, T., ... \& Wynn, M. (2011, August). Process mining manifesto. In International Conference on Business Process Management (pp. 169-194). Springer, Berlin, Heidelberg.

Van Der Aalst, W. (2012). Process mining: Overview and opportunities. ACM Transactions on Management Information Systems (TMIS), 3(2), 1-17.

Werner, M. (2017). Financial process mining-Accounting data structure dependent control flow inference. International Journal of Accounting Information Systems, 25, 57-80. 